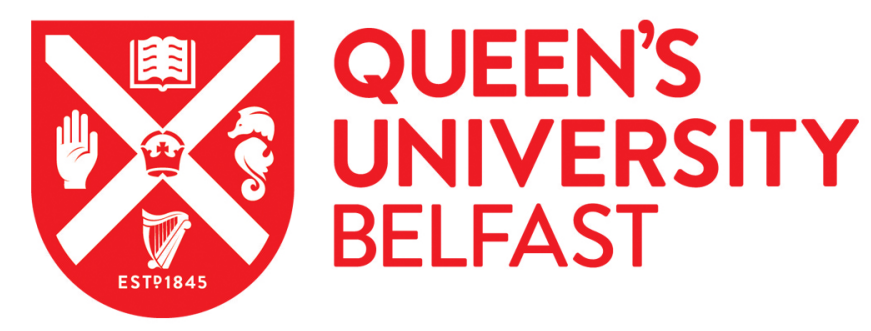

\title{
Optically controlled dense current structures driven by relativistic plasma aperture-induced diffraction
}

Gonzalez-lzquierdo, B., Gray, R. J., King, M., Dance, R. J., Wilson, R., McCreadie, J., Butler, N. M. H., Capdessus, R., Hawkes, S., Green, J. S., Borghesi, M., Neely, D., \& McKenna, P. (2016). Optically controlled dense current structures driven by relativistic plasma aperture-induced diffraction. Nature Physics, 12, 505-512. https://doi.org/10.1038/nphys3613

Published in:

Nature Physics

Document Version:

Peer reviewed version

Queen's University Belfast - Research Portal:

Link to publication record in Queen's University Belfast Research Portal

Publisher rights

(C) 2016 Macmillan Publishers Limited

\section{General rights}

Copyright for the publications made accessible via the Queen's University Belfast Research Portal is retained by the author(s) and / or other copyright owners and it is a condition of accessing these publications that users recognise and abide by the legal requirements associated with these rights.

Take down policy

The Research Portal is Queen's institutional repository that provides access to Queen's research output. Every effort has been made to ensure that content in the Research Portal does not infringe any person's rights, or applicable UK laws. If you discover content in the Research Portal that you believe breaches copyright or violates any law, please contact openaccess@qub.ac.uk. 


\title{
Optically controlled dense current structures driven by relativistic plasma aperture-induced diffraction
}

\author{
B. Gonzalez-Izquierdo, ${ }^{1}$ R. J. Gray, ${ }^{1}$ M. King, ${ }^{1}$ R. J. Dance, ${ }^{1}$ \\ R. Wilson, ${ }^{1}$ J. McCreadie, ${ }^{1}$ N. M. H. Butler, ${ }^{1}$ R. Capdessus, ${ }^{1}$ S. \\ Hawkes, ${ }^{2}$ J. S. Green, ${ }^{2}$ M. Borghesi, ${ }^{3}$ D. Neely, ${ }^{2,1}$ and P. McKenna ${ }^{1, *}$ \\ ${ }^{1}$ SUPA Department of Physics, University of Strathclyde, Glasgow G4 0NG, UK \\ ${ }^{2}$ Central Laser Facility, STFC Rutherford Appleton Laboratory, Oxfordshire OX11 0QX, UK \\ ${ }^{3}$ Centre for Plasma Physics, Queens University Belfast, Belfast BT7 1NN, UK
}

\begin{abstract}
The collective response of charged particles to intense fields is intrinsic to plasma accelerators and radiation sources, relativistic optics and many astrophysical phenomena. Here we show that the fundamental optical process of diffraction of intense laser light occurs via the self-generation of a relativistic plasma aperture in thin foils undergoing relativistic induced transparency. The plasma electrons collectively respond to the resulting near-field diffraction pattern, producing a beam of energetic electrons with spatial structure which can be controlled by variation of the laser pulse parameters. It is shown that static electron beam, and induced magnetic field, structures can be made to rotate at fixed or variable angular frequencies depending on the degree of ellipticity in the laser polarization. The concept is demonstrated numerically and verified experimentally. It is a viable step towards optical control of charged particle dynamics in laser-driven sources.
\end{abstract}

\footnotetext{
*Electronic address: paul.mckenna@strath.ac.uk
} 
The formation of current structures due to the collective response of charged particles to a perturbation is one of the most fundamental properties of plasma. This is manifest in plasma dynamics ranging from flares and X-ray jets on the sun to disruptive instabilities in fusion plasmas. This feature is also exploited to great effect in the development of compact laser-based particle accelerators and radiation sources, which have wide-ranging potential applications in science, medicine and industry. Controlling the collective motion of plasma electrons in response to perturbation produced by intense laser light is key to the development of these novel sources. Pertinent examples in plasma with density low enough for laser light to propagate (underdense plasma) include the self-generated plasma cavity or 'bubble' produced in laser-driven wakefield acceleration [1] and plasma channels [2]. These structures are formed principally by the ponderomotive force induced by the propagating laser pulse, which expels electrons from the regions of high laser intensity, and by self-generated fields induced by the current displacement [3]. Shaping the spatial-intensity profile of a laser focus enables control over the collective electron motion and thereby the properties of the beams of high energy particles and radiation produced. In the case of wakefield acceleration, for example, the laser focal spot size is matched to the size of the plasma bubble as defined by the characteristic plasma oscillation frequency in order to optimize the acceleration [4]. For ultrashort pulses the degree of control on the intensity profile is typically limited to variation of the laser focal spot size by changing the F-number of the focusing optic or by using a deformable mirror to control the laser wavefront.

In the case of solid targets used for ion acceleration $[5,6]$ and high harmonic generation $[7,8]$, the laser light can propagate only to the region of the critical density at which the plasma frequency is equal to the laser light frequency. At the peak laser intensities achievable at present, plasma electrons oscillate at relativistic velocities such that an ultrathin foil undergoing expansion becomes relativistically transparent to the laser pulse [9-14]. The physics underpinning relativistic induced transparency (RIT) in thin foils is discussed by Vshivkov et al [15]. It has been shown that the effect can modify the rising edge profile, duration and polarization of the laser pulse [16-19] and that it is important in driving new ion acceleration $[20,21]$ and radiation production [22-24] mechanisms.

For the first time, we demonstrate that an ultraintense laser pulse induces a 'relativistic plasma aperture' in a thin foil and as a result undergoes the fundamental optic 
process of diffraction. It is demonstrated, both numerically and experimentally, that the collective electron motion (including angular frequency of rotation) is determined by the resulting near-field diffraction pattern and can be controlled by simply varying the polarization of the laser. This new insight into relativistic transparency and charged particle dynamics is important for a wide variety of applications, including ion and radiation source development. It is also shown that helical magnetic fields can be induced using this concept, which could potentially be used in laboratory investigations of similar field structures in astrophysical jets.

\section{NEAR-FIELD DIFFRACTION DURING RELATIVISTIC INDUCED TRANSPARENCY}

The interference of waves which encounter a pinhole (or other obstacle) that is comparable in size to the wavelength gives rise to diffraction phenomenon according to the Huygens-Fresnel principle $[25,26]$. In the case of a thin foil target undergoing RIT, Vshivkov et al [15] derive the conditions for relativistic transparency as $a_{o}>>\omega_{p}^{2} \ell / 2 c \omega_{L}$, where $\omega_{L}$ and $\omega_{p}$ are the laser and plasma frequency, respectively, $\ell$ is the target thickness and $a_{o}$ is the light amplitude. During the interaction, a region of the target near the peak of the focused intensity for which $n_{e}<n_{c}^{\prime}$ becomes relativistically underdense. Here, $n_{e}$ is the plasma electron density and $n_{c}^{\prime}=\gamma m_{e} \epsilon_{0} \omega_{L}^{2} / e^{2}$ is the relativistically corrected critical plasma density $\left(\gamma\right.$ is the Lorentz factor, $m_{e}$ is the electron rest mass and $\epsilon_{0}$ is the vacuum permittivity). The diameter of this circular region, shown schematically in Fig. 1a for a Gaussian laser pulse, depends on the intensity profile of the focal spot and the plasma expansion characteristics. It will typically be of the order of the full width at half maximum (FWHM) of the laser focus and therefore $\sim 2-3$ times the laser wavelength for a tightly focused (near diffraction limited) beam. Thus, after formation of this aperture on the pulse rising edge, the conditions are ideal for strong diffraction of the remainder of the laser pulse propagating through it (as will be shown below).

We begin by considering the near-field diffraction pattern for the idealized case of a fixed aperture, i.e. without plasma evolution effects, and linearly polarized light. Figure $1 \mathrm{~b}$ shows 3D PIC simulation results in which a laser pulse with focal spot diameter of $3 \mu \mathrm{m}$ (FWHM) is passed through a fixed $3 \mu \mathrm{m}$ diameter circular aperture. The laser is polarized along the $\mathrm{Y}$-axis and the intensity distribution patterns in the $\mathrm{Y}-\mathrm{Z}$ plane at 
three example $X$ positions are shown in Fig. 1c. The near-field pattern (i.e. at small $\mathrm{X}$ ) is observed to vary strongly with position from an analogous even- $\mathrm{IG}_{20}$ mode (InceGaussian mode with ellipticity 0.1 ) at $\mathrm{X}=0.4 \mu \mathrm{m}$ to the odd- $\mathrm{IG}_{11}$ mode at $\mathrm{X}=1.2 \mu \mathrm{m}$. The pattern diffracts into an even- $\mathrm{IG}_{00}$ mode in the far-field. The distribution is fully described by a vectorial analysis using Hertz Vector Diffraction Theory (HVDT) [27], as shown in Fig. 1d.

Next we replace the fixed aperture target with a uniform thin foil (i.e. initially without aperture) and investigate the laser-generated relativistic plasma aperture, the effect this has on the propagating laser light and in turn how the evolving laser spatial profile influences the beam of fast electrons accelerated forward. This is investigated using full 3D PIC simulations for the same laser pulse and target parameters as the experiment discussed below.

Figure 2a-e shows an example simulation result for the laser intensity and electron density just after RIT occurs. The laser light is again linearly-polarized, along the Y-axis. Note that for all of the figures, the Lorentz factor, $\gamma$, is evaluated locally for each cell in the simulation considering the average electron momentum. Interference between laser light which is reflected from the target before RIT occurs and the remaining incoming laser light results in a standing wave distribution with local nodes and anti-nodes at the target front side $(\mathrm{X}<0)$. Despite local deformation of the target due to laser radiation pressure, during RIT the remainder of the laser pulse diffracts as it passes through the evolving plasma aperture - the evolution of the aperture, including at late times on the falling edge of the laser pulse, is shown in Fig. $2 b$. The resulting diffraction pattern at the target rear (shown in Fig. 2a and c) is very similar, in both the near and far fields, to the HVDT-calculated patterns shown in Fig. 1. Furthermore, the simulation clearly shows that the accelerated electrons respond to the diffracted laser intensity distribution. In Fig. $2 c$, at the $X$ position at which the electron density is highest on axis $(X \sim 1 \mu \mathrm{m})$, the laser profile has a double diffraction lobe distribution orientated perpendicular to the polarization direction, similar to the distribution shown in Fig. 1b, and an Airy disk distribution in the far field $(X>4 \mu \mathrm{m})$, again similar to the far-field pattern in Fig. 1b. The electrons in the region of the double diffraction lobe are subject to a transverse ponderomotive force in the plane of the target, as shown by the hollow black arrows (from field mapping) in Fig. 2e. This drives the electrons into a double lobe distribution, perpendic- 
ular to the laser polarization direction. A smaller number of electrons are also trapped on-axis between the opposing ponderomotive forces of similar magnitude produced by the two laser diffraction nodes.

\section{POLARIZATION SENSITIVITY}

Given that diffraction in the near-field is sensitive to laser polarization, we next consider the extent to which the electron beam spatial distribution can be controlled by changing the polarization. As highlighted above, the results in Fig. 2c-e are for linearly polarized pulses. Corresponding simulation results are presented for elliptical and circular polarization in Fig. 2f-h and Fig. 2i-k, respectively. The results are explained with reference to model calculations of the angular velocity of the polarization vector and magnitude of the electric field as shown in Fig. 2l-n for one laser period. In the linear polarization case the laser electric field flips between the two lobes over each half-laser period, producing the angular asymmetry in the ponderomotive force discussed above. With circularly polarized light, because the phase difference is $\pi / 2$, the field components produce a dynamic intensity profile which makes a complete rotation at constant angular velocity around the laser propagation axis once per laser period. The instantaneous radial ponderomotive force is asymmetric, as shown in Fig. 2k, but when averaged over a laser period (or more) a ring-like electron distribution centred on the laser propagation axis is produced. The elliptical polarization ( $\pi / 4$ ellipticity) case exhibits elements of both types of behaviour. The polarization vector rotates, but with an angular velocity which varies over the laser period. The magnitude of the laser electric field is highest during the part of the laser period where the polarization vector rotates slowest, as seen when comparing the red phases of the curves in Fig. 2l-n. The plasma electrons respond to the angular variation in the radial ponderomotive force to produce a double lobe orientated perpendicular to the 'average' polarization axis (the red parts of the circle in Fig. $2 \mathrm{~h}$ correspond to the red phases of the curves in Fig. 2l-n).

We note that the use of circularly polarized light results in significantly less electron heating and expansion and consequently more radiation-pressure-driven target deformation. RIT therefore occurs later in the interaction, producing a smaller aperture compared to the linear and elliptical cases. 
The rotating laser diffraction structure instigates an in-phase rotation of a structure in the plasma electron density as shown in the time-resolved results in Fig. 3. The structure consists of a main high energy component rotating with a small radius of curvature about the propagation axis, with a lower density halo of lower energy electrons, $\pi / 2$ out-ofphase. The structure continues to rotate after the laser pulse has decayed, at $\sim 120 \mathrm{fs}$, as shown in Fig. 3c,d.

The behaviour predicted in these simulations was tested experimentally using the Gemini laser [28], which delivers light with a central wavelength of $800 \mathrm{~nm}$ in pulses with $40 \mathrm{fs}$ FWHM duration. The peak laser intensity for these measurements was $6 \times 10^{20}$ $\mathrm{Wcm}^{-2}$ and the targets were $10 \mathrm{~nm}$-thick Al. The salient features of the results are demonstrated in Fig. 4, which shows example time-integrated measurements of the electron beam above given energy thresholds, measured in the $\mathrm{Y}-\mathrm{Z}$ plane, for all three polarization cases. The results are in good agreement with 3D EPOCH simulation results integrated over 5 laser cycles at the end of the pulse. As the measurements are made $3 \mathrm{~cm}$ downstream from the target, the comparison is made in terms of electron angular deflection (as sampled at $\mathrm{X}=6 \mu \mathrm{m}$ in the simulations). From the discussion above, the circularly polarized case may be expected to produce an electron density ring due to the constant rotational velocity of the electric field as shown in Fig. 2l-n. However, the simulations show that in fact there is a slight distortion in the polarization induced by the evolving plasma aperture, resulting in a ring-like distribution with localized maxima.

The experiment results are presented in Fig. 4 for energies above four given thresholds, as set by filters. These measurements verify the behaviour observed in the simulations for all three polarization cases, example results of which are also shown in Fig. 4 for given energy ranges. Note that it is not possible to compare absolute electron densities and therefore it is the shape of the distributions and how they vary with energy which is compared - it is this property which is sensitive to the near-field diffraction pattern. The measured double-lobe electron density feature matches the simulation predictions in terms of the angular separation of the lobes and their orientation with respect to the polarization axes for both the linear and elliptical cases. The larger measured angular width of the lobes may result from space-charge spreading of the beam as it propagates downstream towards the detector. The measurements for circularly-polarized light reproduce both the small-radius central ring with local maxima (labelled 1) and the lower 
density $\pi / 2$ out-of-phase distributions at larger radii (labelled 2) in Fig. 4c for electrons with energy greater than $3.5 \mathrm{MeV}$. The corresponding circular density profile for electrons with energies above $\sim 10 \mathrm{MeV}$ is also reproduced experimentally, as shown in Fig. $4 f$.

We note that a double lobe electron density structure was observed in 3D simulations of ion acceleration in relativistically transparent thin foils by Yin et al [29], using linearly polarized laser light. In the analytical theory presented in that paper, the asymmetric double lobe effect is attributed to the plasma response to the radially symmetric laser intensity profile, and specifically to the combination of oscillatory and nonoscillatory electron momentum components in the plane perpendicular to the laser propagation direction. We have previously reported an experimental observation of a similar double lobe structure, but again only for linearly polarized light [30]. By investigating the sensitivity of the electron distribution to the drive laser polarization, numerically and experimentally, the present study reveals that the underpinning physics giving rise to the lobe features arises from diffraction. This is further confirmed by simulations discussed below in which the relativistic plasma aperture diameter is varied.

\section{ELECTRON SPATIAL PROFILE DEPENDENCE ON APERTURE SIZE}

The relativistic plasma aperture diameter is defined by the condition $n_{e}<n_{c}^{\prime}$ and increases with peak laser intensity for a given target material and thickness. Figure 5a-c and d-f shows linear polarization (Y-axis) simulation results with the laser focus diameter (FWHM) equal to $1.5 \mu \mathrm{m}$ and $6 \mu \mathrm{m}$, respectively. These results are directly comparable to the $3 \mu \mathrm{m}$ case in Fig. 2a-e (the peak laser intensity and duration and target parameters are the same). The smaller aperture case results in a single laser diffraction node, stretched in the polarization direction (Y-axis), which ponderomotively expels electrons preferentially in both directions in $Z$, producing a double lobe electron density distribution. Unlike the $3 \mu \mathrm{m}$ case, because a single laser node is produced on-axis there isn't a secondary low density axial electron population produced.

With increasing laser focal spot size the number of maxima in the near-field diffrac-

tion in the vicinity of the electrons increases. As shown in the supplementary data, the diffraction pattern evolves over time and more fringes are produced because the plasma 
aperture size increases with laser intensity (the condition $n_{e}<n_{c}^{\prime}$ is met over a larger area of the laser focal spot). The expected diffraction pattern is approximately circular and this is observed in the simulation at early times when the plasma aperture is small. With increasing laser intensity, plasma electrons are swept from side to side along the axis of polarization by the laser E-field [31, 32], giving rise to the striped pattern observed in Fig. 5f. This anisotropic plasma response in turn modifies the laser distribution to form stripes interweaved with the electron stripes, as shown in Fig. 5e. The stripes are in fact slightly curved, consistent with a small rotation of the diffraction pattern. An examination of the laser polarization at different points along the $\mathrm{X}$-axis shows that it changes from linear to slightly elliptical when transmitted through the plasma aperture. Anisotropy induced in the heating of the target electrons by a linearly polarized pulse can cause transient effects such as a difference in phase velocities in the two orthogonal directions, which can induce a degree of ellipticity in the polarization [19]. This effect accounts for the slight rotation in the diffraction pattern.

\section{RESULTANT MAGNETIC FIELD STRUCTURES AND RELEVANCE TO LABORATORY AS-}

\section{TROPHYSICS}

Rotating diffraction-driven fast electron patterns can give rise to interesting and potentially useful magnetic field structures. The magnetic field driven by the collective electron response to diffraction of circularly polarized light is helical in structure, tightly precessing at a radius close to that of the plasma aperture $(0.75 \mu \mathrm{m})$, as shown in Fig. 6a. When compared to the magnetic field generated by a lower intensity (but otherwise identical) circularly polarized laser, propagating through a comparable fixed circular aperture of $0.75 \mu \mathrm{m}$ radius (i.e. no plasma; Fig. $6 \mathrm{~b}$ ), it is clear that the diffraction effects play a key role in shaping the magnetic field structure. In both cases the field is shown $30 \mathrm{fs}$ after the peak laser intensity has propagated through the aperture. The laser pulse propagating through the fixed aperture produces a magnetic field which is strongest close to the aperture, reducing as the beam expands. By contrast, in the relativistic plasma aperture case the magnetic field is strong over a longer range and is more helical, suggesting that the field is modified by the induced plasma current structure. The underlying physics of the collective plasma effects on the magnetic field will be the 
subject of future investigation.

The current and field structures produced in this way could be used to generate laboratory analogues of astrophysical phenomena. Similar magnetic field structures have been detected in jets originating from active galactic nuclei, such as 3C 273 [33]. Such structures are commonly identified as being generated from the rotation of the accretion disk, whilst the analogous structures seen here arise from the rotation of the diffraction pattern. Further similarities to these jets can be observed in that the highest momentum component of the accelerated electrons occur along the central laser axis. Surrounding this region, the electron momentum reduces, as seen in Fig. 3d, resulting in an electron beam with properties similar to that of the spine-sheath jet morphology [34].

To test the scaling ratio of the two systems, it can be assumed that the length of the base of an active galactic nucleus (AGN) astrophysical jet is on the order of 1 pc [35]. Similarly, the diffraction-induced helical field is observed numerically over $15 \mu \mathrm{m}$ after the target, giving a length scale ratio of $\sim 2 \times 10^{21}$ between the two systems. It can also be assumed that the jet expands at a velocity close to $c$, resulting in a time scale of $\sim 1 \times 10^{8}$ s. As the laser-accelerated electrons expand at approximately the same velocity, the time scale ratio should correspond to that of the length scale ratio [36]. This would give an interaction time-scale of $\sim 50 \mathrm{fs}$, which is very close to that of the laser pulse duration.

In the sub-parsec region of such astrophysical jets, the magnetic field strength is estimated to be on the order of $1 \times 10^{-3} \mathrm{~T}$ [37] and that the number density can be estimated at between $1 \times 10^{10}$ and $1 \times 10^{16} \mathrm{~m}^{-3}$ [38], giving $\omega_{e} / \Omega_{e} \sim 0.031-31.1$, where $\omega_{e}$ and $\Omega_{e}$ are the electron plasma frequency and cyclotron frequency, respectively. For the plasma aperture diffraction case using a circularly polarized pulse, it is found that $\omega_{e} / \Omega_{e} \sim 0.33$ (which would require $n_{e} \sim 1 \times 10^{12} \mathrm{~m}^{-3}$ for the astrophysical jet). The helical pitch in these simulations increases linearly from $15^{\circ}$ to $55^{\circ}$ over a distance of $15 \mu \mathrm{m}$ from the target. Similarly, the magnetic field produced from these astrophysical jets has been observed to show evidence of an increase in pitch as it propagates further from the source [33]. These various estimates suggest that while there is a huge difference in scale between the two systems, there may be sufficient similarities that would allow future laboratory testing of the formation of helical magnetic field structures in astrophysical jets. 


\section{OUTLOOK}

The present work is the first demonstration that intense laser light can self-generate and be strongly diffracted by a relativistic plasma aperture in thin foils undergoing RIT and that the plasma electrons collectively respond to the resulting near-field diffraction pattern. This is a new phenomenon in relativistic plasma optics and collective electron motion control. It is shown that through suitable choice of laser drive parameters the spatial-intensity distribution of a high current beam of relativistic electrons can be modified and that rotating structures can be induced. It is also shown that the angular rotational frequency can be varied by changing the degree of ellipticity in the laser polarization. This opens up a new direction in spatial and temporal control of electron motion in dense plasma and by extension the evolution of the high fields used to accelerate charged particles and to produce high energy radiation. In thin foils, including those undergoing RIT, the electric fields produced by displacement of electrons accelerate ions to multi-MeV energies. The spatial-intensity profile of the plasma electron beam is effectively mapped into the spatial-intensity profile of the ion beam, as discussed for example in reference [29]. Thus the techniques we introduce to change the profile of the plasma electron beam can also enable the ion beam spatial-intensity distribution to be controlled. Understanding these processes is important to the development of laserdriven ion acceleration, given that many of the most promising schemes involve ultrathin foil targets $[6,20,21]$.

There are a number of other ways in which one could envisage utilizing the plasma aperture concept in particle and radiation source development. Our simulations suggest that it could potentially be used to induce orbital angular momentum states at high laser

intensity. A rotating diffraction lobe structure produced with circularly polarized light results in a helical structure of intense laser light with an optical vortex at the centre, at the beam axis. Such a structure can be used to trap plasma electrons on-axis. A small population of electrons is observed to be trapped between the double lobe structure by the inward directed ponderomotive forces in our linear polarization simulations. This effect could be optimised and used to induce an axial current filament in relatively high density plasma, with potential application to positron and ion acceleration (positively charged particles are attracted towards the electron filament). It has shown in a recent 
theoretical and numerical study involving lower density targets that helical light beams can be used to drive positron acceleration [39]. We further note because the plasma electron motion is induced in phase with the drive laser and that this in turn can be synchronized to a second laser pulse (e.g. by splitting the laser beam), it may be possible to apply this scheme to enhance electron scattering processes in ultraintense laser fields (such as nonlinear Compton or Thomson scattering). Thus, the results are not only of fundamental importance to our understanding of relativistic transparency and the interpretation of experimental results involving transparency in ultrathin foils, but could also profoundly affect the development of laser driven particle and radiation sources involving dense laser-irradiated targets.

\section{METHODS}

\section{Experiment}

The experiment results were obtained using the Gemini [28] Ti:sapphire laser at Rutherford Appleton Laboratory in the UK. The central wavelength is optimized at $800 \mathrm{~nm}$ and the pulse duration was $40 \mathrm{fs}$ (FWHM). The laser beam was focused along target normal onto the front surface of $10 \mathrm{~nm}$-thick aluminium foils, using an off-axis $f / 2$ parabola, to a focal spot diameter of $3 \mu \mathrm{m}$ (FWHM). The laser pulse energy was 2 $\mathrm{J}$, giving a calculated peak intensity of $6 \times 10^{20} \mathrm{Wcm}^{-2}$. A double plasma mirror configuration was used to enhance the intensity contrast to $\sim 10^{11}$ and $\sim 10^{8}$, at 1 ns and 5 ps, respectively, prior to the peak of the pulse. A deformable mirror was used prior to the focusing parabola to ensure a high quality focal spot on target. Thin mica wave plates were used to switch between linear $(\Delta \theta=0)$, elliptical $(\Delta \theta=\pi / 4)$ and circular $(\Delta \theta=\pi / 2)$ polarization, where $\Delta \theta$ is the phase difference between the two orthogonal components of the laser beam. The 2D spatial-intensity distribution of the electron beam was measured $3 \mathrm{~cm}$ downstream from the target using passive stacked layers of Fujifilm imaging plate, interleaved with iron filters to provide energy filtering. The filtering before the first layer is sufficient to prevent the detection of any protons or heavier ions produced. 


\section{Analytical modelling}

The analytical modelling of the diffraction pattern produced by a laser pulse passing through a circular aperture, as shown in Fig. 1, was performed using Hertz Vector Diffraction Theory (HVDT) [27]. With this vectorial model, values for the electromagnetic field can be obtained not only in the plane of the aperture but also downstream. The near-field diffraction pattern, in the region of maximum interaction between the laser and the plasma electrons, is of particular interest here. Although other analytical models like the scalar Rayleigh-Sommerfeld [40] or Fresnel-Kirchhoff [41] models can be used to study diffraction induced by an aperture, they work in the limit that the aperture radius is much bigger than the laser wavelength, that the electric field in the plane of the aperture is known and involve a scalar treatment of the diffracted light. We use the HVDT model to avoid these limitations.

The analytical results reported in Fig. 21-n were obtained by solving the following equations which describe the two orthogonal electric field components for the different

polarizations considered. The orthogonal electric field components are $\overrightarrow{E_{y}}=E_{0 y} \cdot \sin (\phi) \vec{y}$ and $\vec{E}_{z}=E_{0 z} \cdot \sin (\phi-\Delta \theta) \vec{z}$, where $\Delta \theta$ is the phase difference. For linear polarization $\Delta \theta=0$ and $E_{0 z}=0$; for elliptical polarization $\Delta \theta=\pi / 4$ and $E_{0 y}=E_{0 z}$; and for circular polarization $\Delta \theta=\pi / 2$ and $E_{0 y}=E_{0 z}$. The angle with respect to the $+\mathrm{Y}$ axis is $\theta_{\vec{E}_{y} \vec{E}_{z}}=\tan ^{-1}\left(\vec{E}_{z} / \vec{E}_{y}\right)$ and the rotational angular velocity with respect to the $+Y$ axis is $\omega_{R}=d \theta_{\vec{E}_{y}} \vec{E}_{z} / d t$. The magnitude of the total electric field is $\left\|E_{T}\left(\vec{E}_{y}, \vec{E}_{z}\right)\right\|=\sqrt{{\overrightarrow{E_{y}}}^{2}+{\overrightarrow{E_{z}}}^{2}}$.

\section{Simulations}

The simulations are performed using the fully relativistic 3D EPOCH particle-in-cell (PIC) code [42]. The simulation space is defined as a $20 \mu \mathrm{m} \times 20 \mu \mathrm{m} \times 20 \mu \mathrm{m}$ box with $1000 \times 720 \times 720$ computational mesh cells. The laser wavelength is $800 \mathrm{~nm}$. The pulse has a Gaussian temporal profile with $40 \mathrm{fs}$ width (FWHM) focused to a Gaussian intensity distribution of $3 \mu \mathrm{m}$ FWHM. The peak laser intensity is $6 \times 10^{20} \mathrm{Wcm}^{-2}$. Simulations are performed with the laser pulse polarized linearly (p-polarization), elliptically (ellipticity of $\pi / 4$ ) or circularly. The simulations are run for a total duration of $200 \mathrm{fs}$ to ensure full propagation of the laser pulse through the simulation space. 
The target is representative of a solid $10 \mathrm{~nm}$-thick $\mathrm{Al}^{13+}$ slab with $6 \mathrm{~nm}$-thick $\mathrm{C}^{6+}$ and $\mathrm{H}^{+}$mixed contamination layers (assumed to be a hydrocarbon with the form $\mathrm{C}_{2} \mathrm{H}_{6}$ ) on the front and rear surfaces, all fully ionized. The smallest mesh cell size that we can use in these computationally intensive 3D simulations is $20 \mathrm{~nm}$ (the computation time exponentially increases with the reduction in mesh size and each simulation requires 22 thousand core-hours). The target is thus pre-expanded to a Gaussian profile (with 245 nm FWHM) in order to have a sufficient number of cells across it to avoid self-heating and other numerical artefacts. This gives reduced peak electron densities of $\sim 14.3 n_{c}$, for the main target and $\sim 3.7 n_{c}$ for the contamination layers. The ion density is initialized to neutralize the electrons using appropriate proportions of $\mathrm{Al}^{13+}, \mathrm{C}^{6+}$ and $\mathrm{H}^{+}$ions. This approach is justified given that experimentally some degree of expansion, driven by electron heating early on the rising edge of the laser pulse, will occur in order to enable RIT to take place.

The initial electron temperature is chosen to be low enough to avoid artificial thermal induced effects, but high enough to resolve the Debye length as closely as possible. Simulations at increased temperatures fully resolving the Debye length do not significantly change the physics reported here but exhibit thermal instabilities on the target surface away from the laser focus. Initially there are $\sim 22$ simulation particles per cell per species (total of $3.11 \times 10^{9}$ simulation particles). The code assumes no binary collisions and the boundaries are defined as free-space. Although there is a degree of particle loss and charge build-up at the boundaries, this is acceptable as the region of interest is suitably far from any boundary effects given the overall simulation runtime.

\section{REFERENCES}

[1] Pukhov, A. \& Meyer-ter-Vehn, J. Laser wake field acceleration: the highly non-linear brokenwave regime. Appl. Phys. B 74, 355-361 (2002)

[2] Pukhov, A. \& Meyer-ter-Vehn, J. Relativistic magnetic self-channeling of light in near-critical plasma: three-dimensional particle-in-cell simulation. Phys. Rev. Lett. 76, 3975 (1996)

[3] Kaluza M.C. et al. Measurement of magnetic-field Structures in a laser-wakefield accelerator. Phys. Rev. Lett. 105, 115002 (2010) 
[4] Thomas A.G.R. et al. Effect of laser-focusing conditions on propagation and monoenergetic electron production in laser-wakefield accelerators. Phys. Rev. Lett. 98, 095004 (2007)

[5] Daido H. et al. Review of laser-driven ion sources and their applications. Rep. Prog. Phys. 75, 056401 (2012)

[6] Macchi A. et al. Ion acceleration by superintense laser-plasma interaction. Rev. Mod. Phys. 85, $751(2013)$

[7] Ganeev R.A. High-order harmonic generation in a laser plasma: a review of recent achievements. J. Phys. B: At. Mol. Opt. Phys 40, R213 (2007)

[8] Dromey B. et al. High harmonic generation in the relativistic limit. Nature Phys. 2, 456-459 (2006)

[9] Guerin S. et al. Propagation of ultraintense laser pulses through overdense plasma layers. Phys. Plasmas 3, 2693 (1996)

[10] Fuchs J. et al. Transmission through highly overdense plasma slabs with a subpicosecond relativistic laser pulse. Phys. Rev. Lett. 80, 2326 (1998)

[11] Cattani F. et al. Threshold of induced transparency in the relativistic interaction of an electromagnetic wave with overdense plasmas. Phys. Rev. E. 1234, (2000)

[12] Tushentsov M. et al. Electromagnetic energy penetration in the self-induced transparency regime of relativistic laser-plasma interactions. Phys. Rev Lett. 87, 275002 (2001)

[13] Willingale L. et al. Characterization of high-intensity laser propagation in the relativistic transparent regime through measurements of energetic proton beams. Phys. Rev. Lett. 102, 125002 (2009)

[14] Eremin V.I. et al. Relativistic self-induced transparency effect during ultraintense laser interaction with overdense plasmas: why it occurs and its use for ultrashort electron bunch generation. Phys. Plasmas 17, 043102 (2010)

[15] Vshivkov V.A. et al. Nonlinear electrodynamics of the interaction of ultra-intense laser pulses with a thin foil. Phys. Plasmas 5, 2727 (1998)

[16] Reed S.A. et al. Relativistic plasma shutter for ultraintense laser pulses. Appl. Phys. Lett. 94, 201117 (2009)

[17] Wang H.Y. et al. Laser shaping of relativistic intense, short Gaussian pulse by a plasma lens. Phys. Rev. Lett. 107, 265002 (2011)

[18] Palaniyappan S. et al. Dynamics of relativistic transparency and optical shuttering in ex- 
panding overdense plasmas. Nature Phys. 8, 11 (2012)

[19] Stark. D.J. et al. Relativistic plasma polarizer: impact of temperature anisotropy on relativistic transparency. Phys. Rev. Lett. 115, 025002 (2015)

[20] Henig. A. et al. Enhanced laser-driven ion acceleration in the relativistic transparency regime. Phys. Rev. Lett. 103, 045002 (2009)

[21] Macchi A. et al. "Light Sail" acceleration reexamined. Phys. Rev. Lett. 103, 085003 (2009)

[22] Dromey B. et al. Coherent synchrotron emission from electron nanobunches formed in relativistic laser-plasma interactions. Nature Phys. 8, 11 (2012)

[23] Kiefer D. et al. Relativistic electron mirrors from nanoscale foils for coherent frequency upshift to the extreme ultraviolet. Nat. Commun. 4, 1763 (2013)

[24] Yeung M. et al. Dependence of laser-driven coherent synchrotron emission efficiency on pulse ellipticity and implications for polarization gating. Phys. Rev. Let. 112, 123902 (2014)

[25] Fresnel A. Memoire sur la Diffraction de la Lumiere Ann. Chim et Phys. 1, 129 (1816)

[26] Stratton, J.A. \& Chu, L.J. Diffraction theory of electromagnetic waves. Phys. Rev. 56, 99-107 (1939)

[27] Guha, S. \& Gillen, G. Description of light propagation through a circular aperture using nonparaxial vector diffraction theory. Opt. Express 13, 1424-1447 (2005)

[28] Hooker C.J. et al. The Astra Gemini project - A dual-beam petawatt Ti:Sapphire laser system. J. Phys. IV France 133, 673-677 (2006)

[29] Yin, L. et al. Three-Dimensional dynamics of breakout afterburner ion acceleration using high-contrast short-pulse laser and nanoscale targets. Phys. Rev. Lett. 107, 045003 (2011)

[30] Gray, R.J. et al. Azimuthal asymmetry in collective electron dynamics in relativistically transparent laser-foil interactions. New J. Phys. 16, 093027 (2014)

[31] Sgattoni, A. et al. Laser-driven Rayleigh-Taylor instability: Plasmonic effects and threedimensional structures. Phys. Rev. E 91, 013106 (2015)

[32] Tamburini, M. et al. Radiation-pressure-dominant acceleration: Polarization and radiation reaction effects and energy increase in three-dimensional simulations. Phys. Rev. E 85, 016407 (2012)

[33] Asada K. et al. A helical magnetic field in the jet of 3C 273. Publ. Astron. Soc. Japan 54,(3) L39-L43 (2002)

[34] Ghisellini G. et al. Structured jets in TeV BL Lac objects and radiogalaxies. Astron. Astrophy. 
432, 401-410 (2005)

[35] Zamaninasab, M., Clausen-Brown, E., Savolainen, T., Tchekhovskoy, A., Dynamically important magnetic fields near accreting supermassive black holes, Nature 510, 126-128 (2014)

[36] Bouquet S. et al. From lasers to the universe: Scaling laws in laboratory astrophysics. High Energy Density Physics 6,(4) 368-380 (2010)

[37] Marti-Vidal, I., Muller, S., Vlemmings, W., Horellou, C., Aalto, S., A strong magnetic field in the jet base of a supermassive black hole. Science 348, 6232 (2015)

[38] Gomez, J.-L. et al., Flashing Superluminal Components in the Jet of the Radio Galaxy 3C120. Science 289, 5488 (2000)

[39] Corde, S. et al., Multi-gigaelectronvolt acceleration of positrons in a self-loaded plasma wakefield. Nature 524, 442-445 (2015)

[40] Rayleigh, J.W. On the passage of waves through apertures in Plane screens, and allied problems. Philos. Mag. 43, 259-272 (1897)

[41] Kirchhoff, G.R. Zur theorie der lichtstrahlen. Ann. Phys. 254, 663-695 (1883)

[42] Brady, C.S. and Arber, T.D. An ion acceleration mechanism in laser illuminated targets with internal electron density structure. Plasma Phys. Control. Fusion 53, 015001 (2001)

\section{ACKNOWLEDGMENTS}

We thank the Gemini team at the Central Laser Facility of the Rutherford Appleton Laboratory for their support during the experiment; in particular, N. Booth and D. Symes. We acknowledge the use of the ARCHIE-WeST and ARCHER high performance computers. This work is supported by EPSRC (grants: EP/J003832/1, EP/L001357/1, $\mathrm{EP} / \mathrm{K} 022415 / 1$ and EP/L000237/1), STFC (grant number ST/K502340/1) and the US Air Force Office of Scientific Research (grant: FA8655-13-1-3008). EPOCH was developed under EPSRC grant EP/G054940/1.

\section{AUTHOR CONTRIBUTIONS}

B.G.-I., R.J.G., M.K. and P.M. conceived the experiment. R.J.G., R.J.D., B.G.-I., R.W., J.M., N.M.H.B., S.H., J.S.G. and P.M. executed the experiment and B.G.-I. and R.J.G. performed the analysis of the experimental data. M.K. and B.G.-I. performed the simulations 
and analysis of the simulation results, with contributions from R.C.. P.M. provided overall supervision of the work, with contributions from D.N. and M.B.. The manuscript was prepared by P.M., B.G.-I., M.K. and R.J.G. with contributions from all authors.

\section{ADDITIONAL INFORMATION}

Data associated with research published in this paper can be accessed by contacting the corresponding author.

\section{COMPETING FINANCIAL INTERESTS}

The authors declare no competing financial interests. 


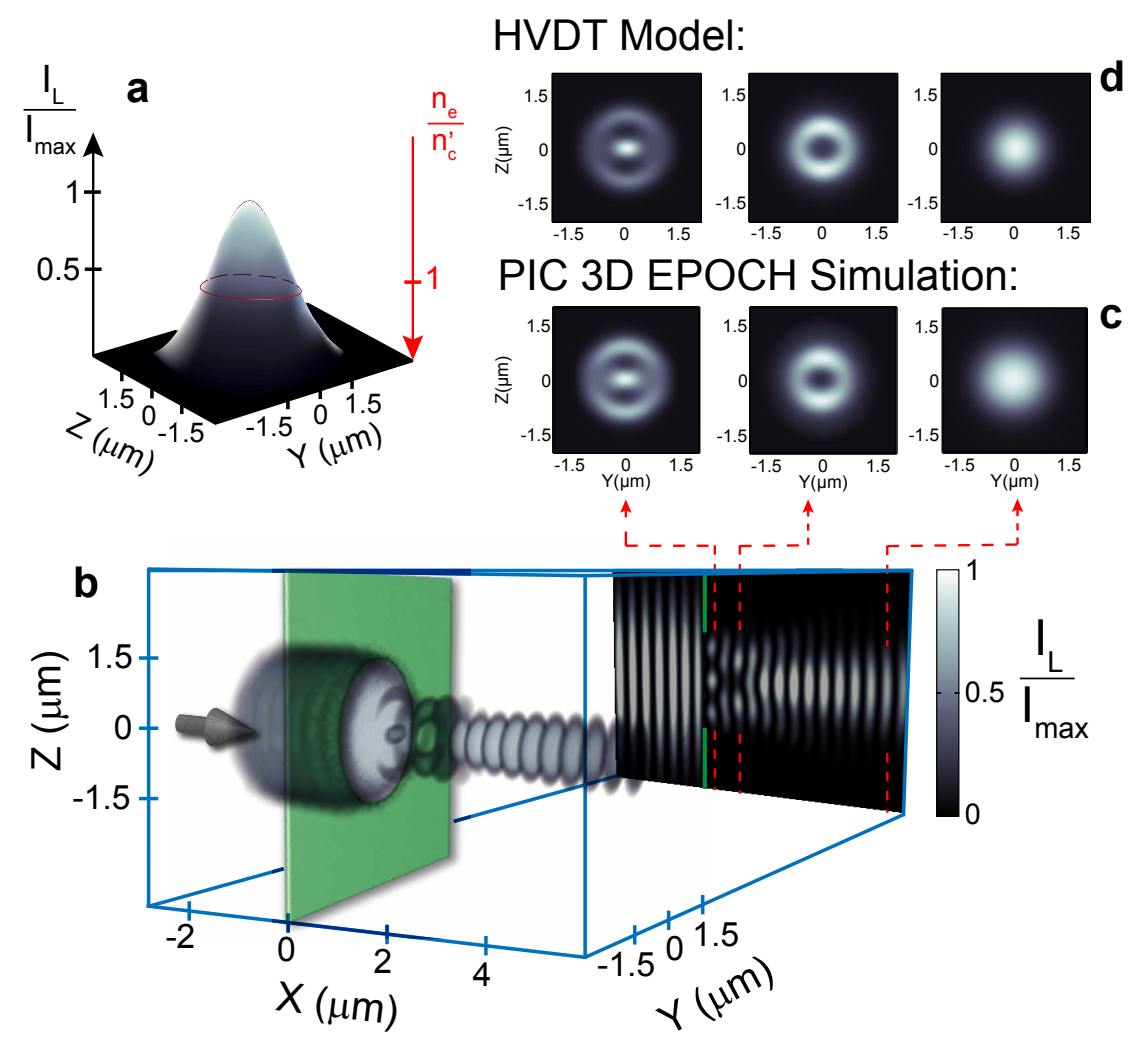

Figure 1: Intensity diffraction pattern induced by a fixed, pre-defined aperture. a, Schematic showing the Gaussian spatial-intensity distribution of an ultraintense laser pulse with diameter (FWHM) equal to $3 \mu \mathrm{m}$. For the case of near-critical, dense plasma, relativistic induced transparency occurs at intensities above those bounded by the ring corresponding to the threshold condition $\left(n_{c}^{\prime}=n_{e}\right)$, enabling this portion of the laser pulse to be transmitted; $\mathbf{b}-\mathbf{c}$, Simulation output showing the spatial-intensity variation of the diffraction pattern of a laser pulse with $3 \mu \mathrm{m}$ (FWHM) diameter passing through a fixed $3 \mu \mathrm{m}$-diameter aperture: $\mathbf{b}, 3 \mathrm{D}$ profile and $2 \mathrm{D}(\mathrm{X}-\mathrm{Z})$ cut-away in the $\mathrm{Y}=0$ plane; $\mathbf{c}, 2 \mathrm{D}$ profile in the plane of the target at $\mathrm{X}=0.4 \mu \mathrm{m}, \mathrm{X}=1.2 \mu \mathrm{m}$ and $\mathrm{X}=5.2 \mu \mathrm{m}$, exhibiting mode structures; $\mathbf{d}$, Calculated diffraction patterns at the same three positions using Hertz vector diffraction theory (HVDT). 

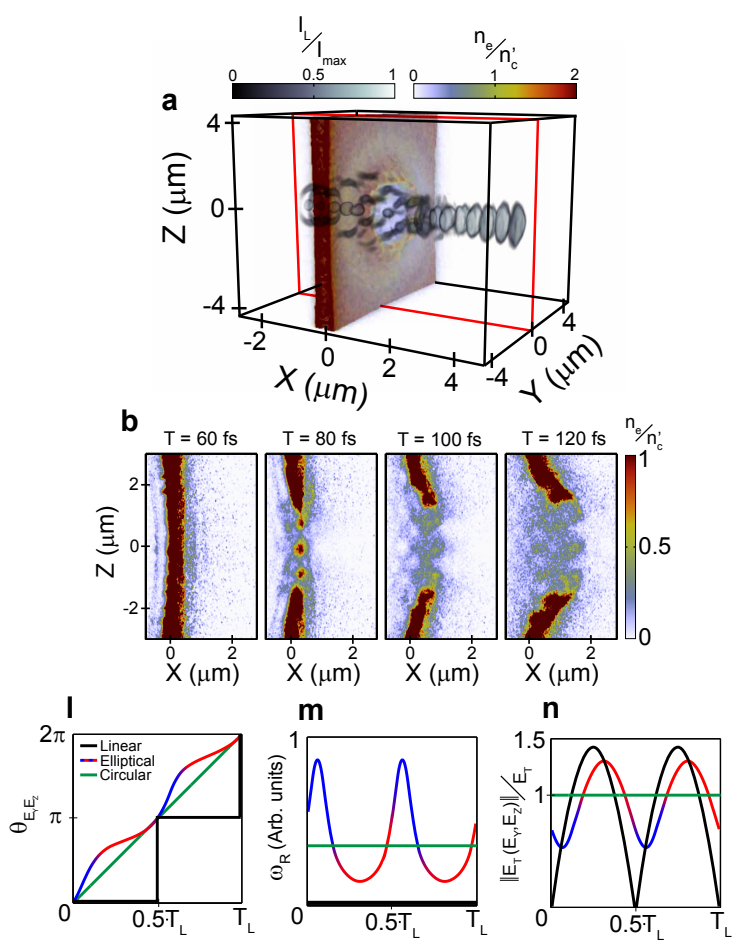
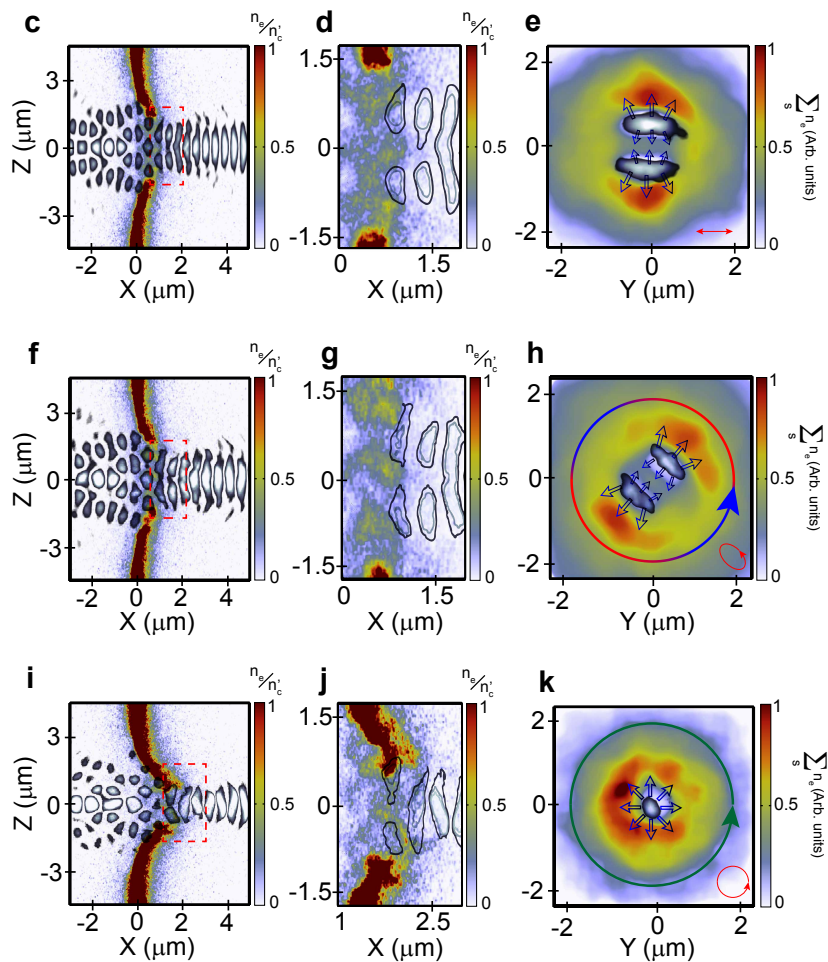

Figure 2: 3D-PIC simulations of laser diffraction and plasma electron density produced by the rela-

tivistic plasma aperture. a, Example simulation result showing the laser intensity, $I$, pattern overlapped with the plasma electron density normalized to $n_{c}^{\prime}$, at $20 \mathrm{fs}$ after RIT occurs. The diffraction pattern is very similar to the fixed aperture case without plasma shown in Fig. 1. The laser is linearly polarized in the $\mathrm{Y}$-axis in both cases. $\mathbf{b}, 2 \mathrm{D}(\mathrm{X}-\mathrm{Z})$ cut-away in the $\mathrm{Y}=0$ plane (red plane highlighted in $\mathbf{a}$ ) showing the electron density at four example time steps. c, 2D (X-Z) cut-away in the $\mathrm{Y}=0$ plane showing the diffraction pattern and electron density. $\mathbf{d}$, Same showing the region of interest bounded by the dashed rectangle in b. The laser is delineated here as a contour to show that the electron distribution is modulated by the laser diffraction pattern. e, 2D (Y-Z) plane showing laser light intensity and electron density integrated over $\mathrm{X}=0.7-1.5 \mu \mathrm{m}$ (corresponding to one laser wavelength in the region of the high density of electrons that are accelerated forward). The hollow black arrows illustrate the direction of the ponderomotive force arising from the gradients in laser intensity. $\mathbf{f}-\mathbf{h}$, Same for elliptically polarized laser light, with the X-Z plane corresponding to the projection of a $45^{\circ}$ rotated plane around the laser propagation axis. $\mathbf{i}-\mathbf{k}$, Same for circularly polarized light. The red arrow inserts indicate the laser polarization. 1-n, Model representation of: 1, angle of the polarization vector with respect to the $+\mathrm{Y}$ axis; $\mathbf{m}$, angular velocity of the polarization vector rotation with respect to the $+\mathrm{Y}$ axis; and $\mathbf{n}$, magnitude of the laser electric field, for all three polarization cases over one laser period, $T_{L}$. The red 'phase' highlights the corresponding region in the rotation of the diffraction pattern shown in $\mathbf{h}$. 

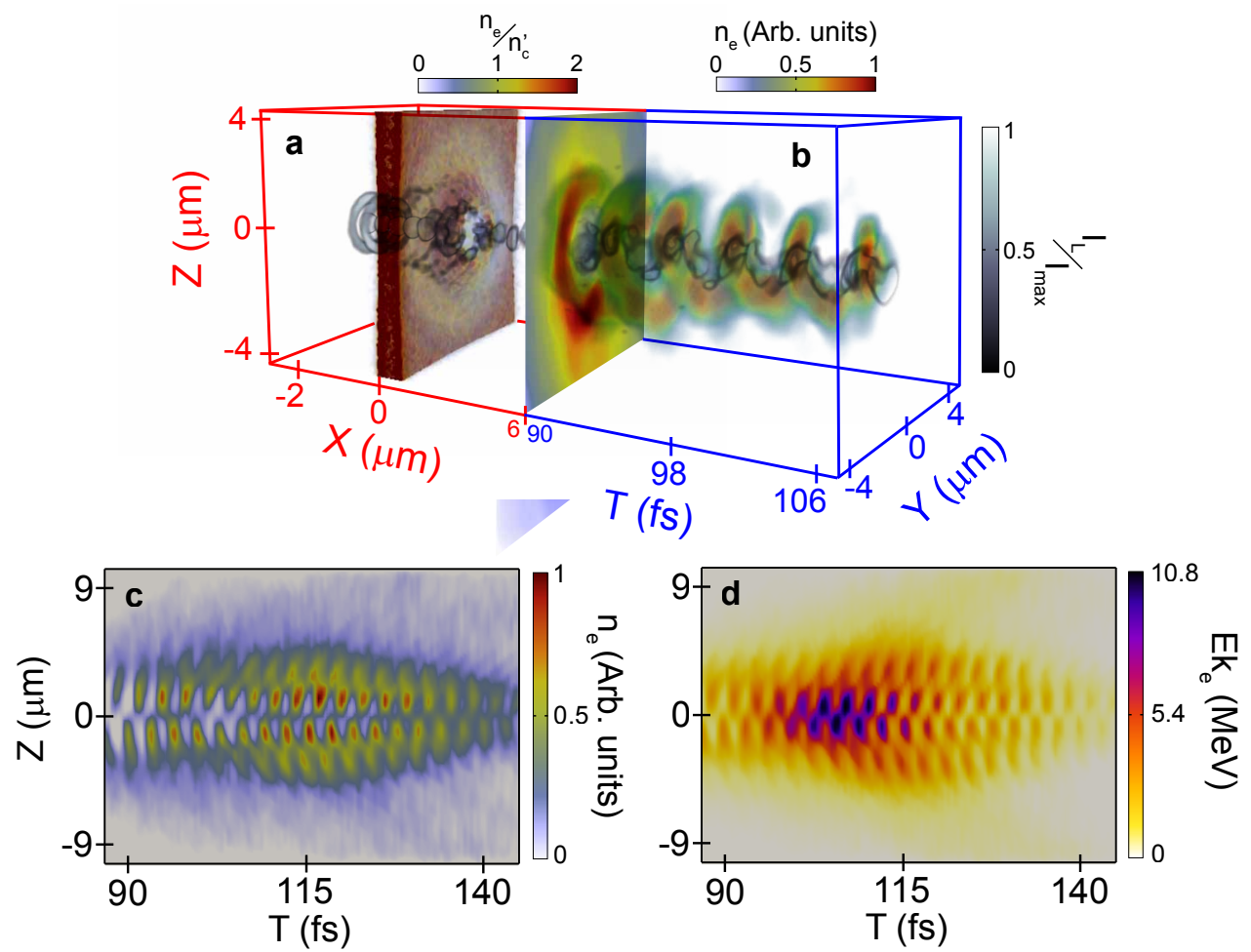

Figure 3: 3D PIC simulation results for circularly polarized light. a-b, Combined plot showing a, the 3D laser intensity profile (up to $\mathrm{X}=6 \mu \mathrm{m}$ ) at $100 \mathrm{fs}$, and $\mathbf{b}$, temporal evolution of the electron density distribution, overlaid with the laser intensity distribution, in the $\mathrm{Y}-\mathrm{Z}$ plane at $\mathrm{X}=6 \mu \mathrm{m}$ from 90 to 107 fs. c, Temporal evolution of the electron density along the $\mathrm{Z}$-axis at $\mathrm{X}=6 \mu \mathrm{m}$ and $\mathrm{Y}=0$. $\mathbf{d}$, Same for electron energy. The electron density and mean kinetic energy in each given cell of the simulation is plotted. The laser diffraction lobe pattern rotates once per laser cycle driving an electron energy and density distribution which rotates in phase with it. The rotational velocity imparted on the electrons drives their rotation after the laser pulse has passed. 


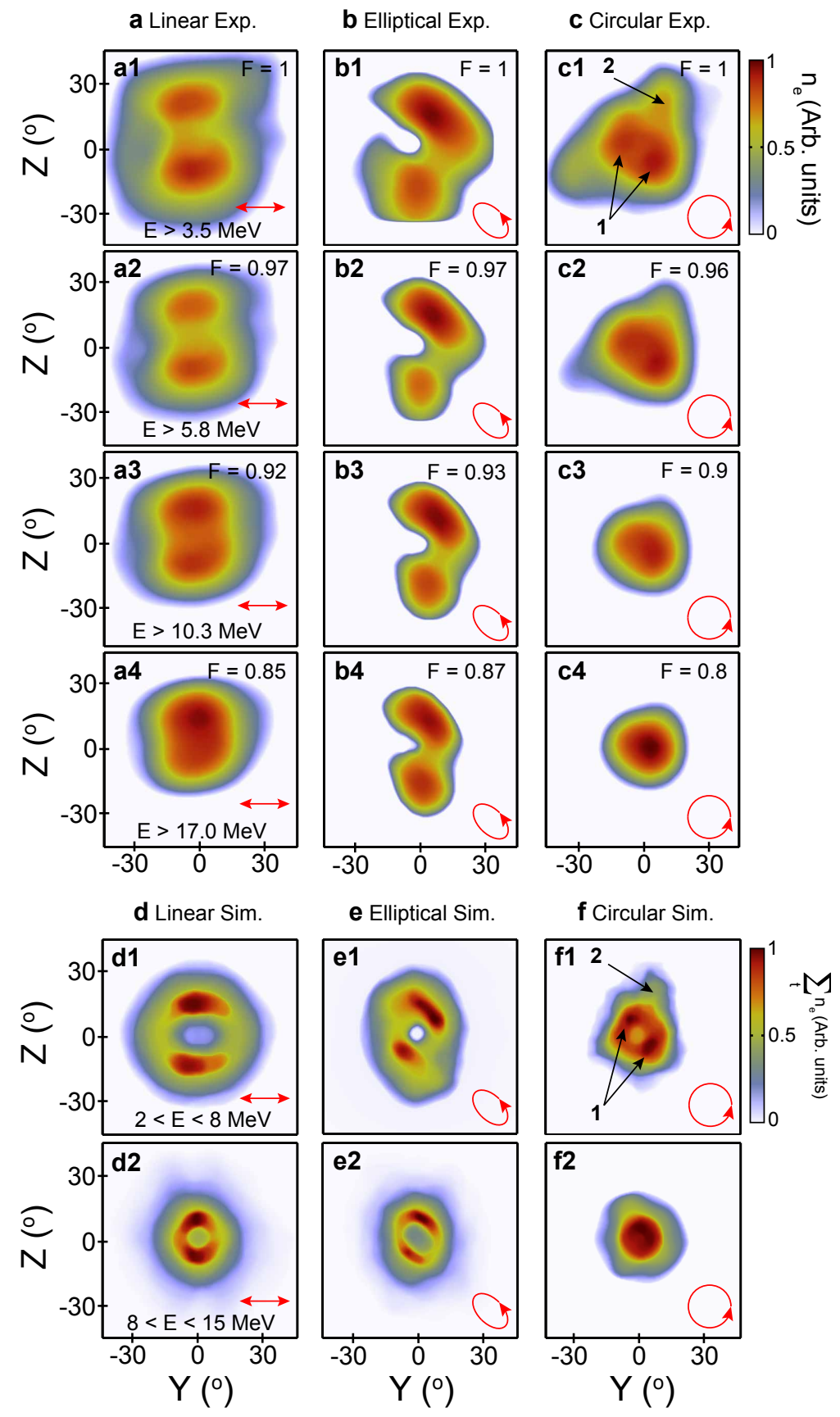

Figure 4: Experiment and 3D PIC simulation results for the electron density distribution. a, Electron density as measured using image plate for electrons with energy greater than: a1, 3.5 MeV; a2, 5.8 MeV; a3, 10.3 MeV; and a4, 17.0 MeV; all for linear polarization. b, Same for elliptical polarization; c, Same for circular polarization. The colour maps are scaled by the stated value $F$ (between 0.8 and 1 ) to clearly show the features of interest at each energy slice. $\mathbf{d}$, Simulated time-integrated electron density in the plane Y-Z at $6 \mu \mathrm{m}$ downstream from the rear side of the target, corresponding to linear polarization for the two stated energy ranges $(\mathbf{d} 1,2<E<8 \mathrm{MeV}$ and $\mathbf{d} 2,8<E<15 \mathrm{MeV})$. e, Same for elliptical polarization; $\mathbf{f}$, Same for circular polarization. The features labelled 1 and 2 in $\mathbf{c} \mathbf{1}$ and $\mathbf{f} \mathbf{1}$ are discussed in the main text. 

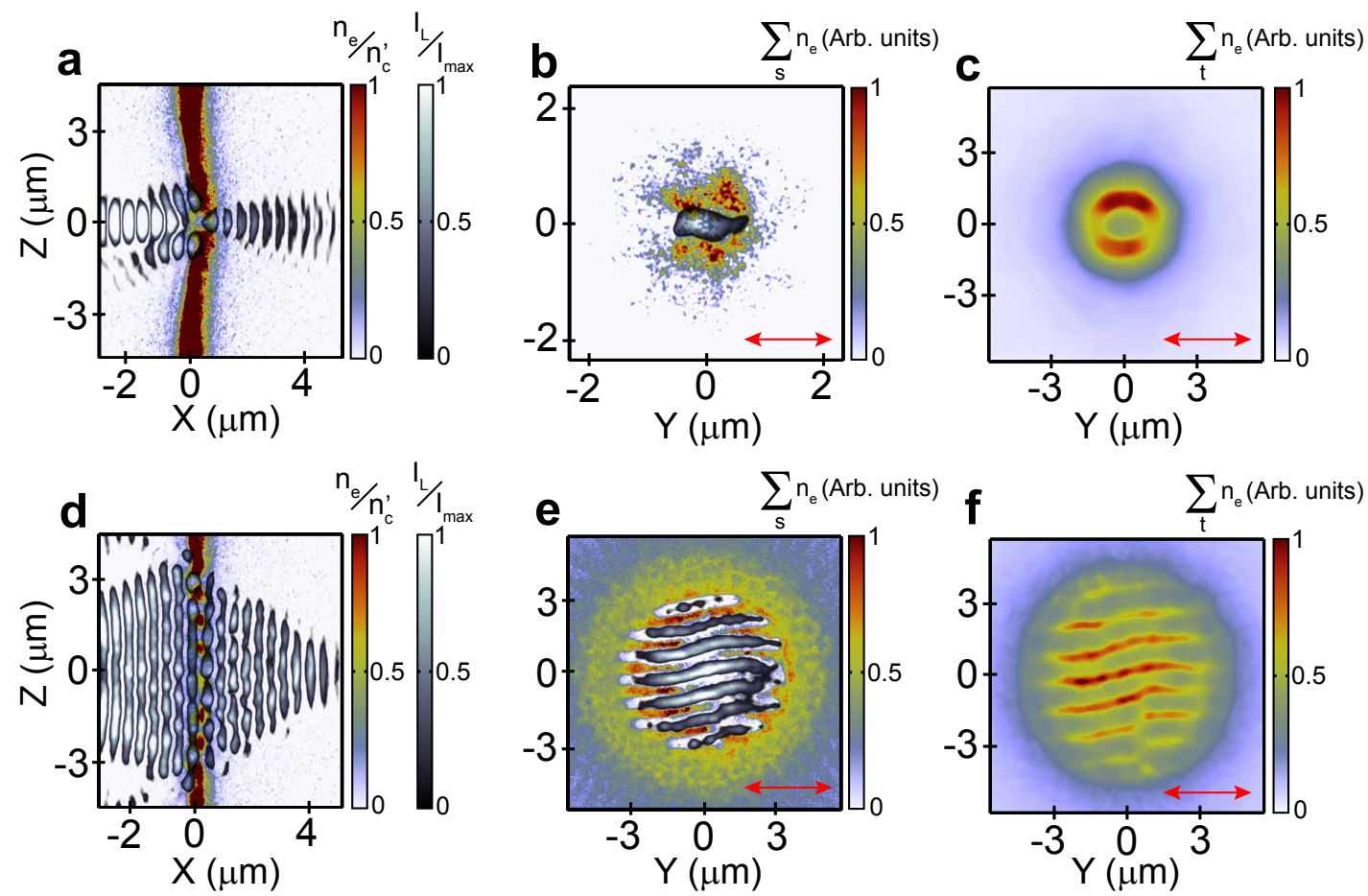

Figure 5: 3D EPOCH simulation with laser focal spot size equal to $1.5 \mu \mathrm{m}$ and $6 \mu \mathrm{m}$. a, Laser intensity and electron density in the X-Z plane at $\mathrm{Y}=0$, for a $1.5 \mu \mathrm{m}$ (FWHM) focal spot. b, Y-Z plane with $n_{e} / n_{c}^{\prime}$ overlapped with the laser intensity profile (both spatially integrated over $\mathrm{X}=0.7-1.5 \mu \mathrm{m}$ ). $\mathbf{c}$, Time integrated electron density in the $\mathrm{Y}-\mathrm{Z}$ plane at $\mathrm{X}=6 \mu \mathrm{m}$. d-f, Same as a-c but with a laser focal spot diameter equal to $6 \mu \mathrm{m}$ (FWHM). In both cases the laser is linearly polarized in the Y-axis. See Supplementary Material for additional discussion of the plasma electron response to diffraction as a function of the plasma aperture size. 


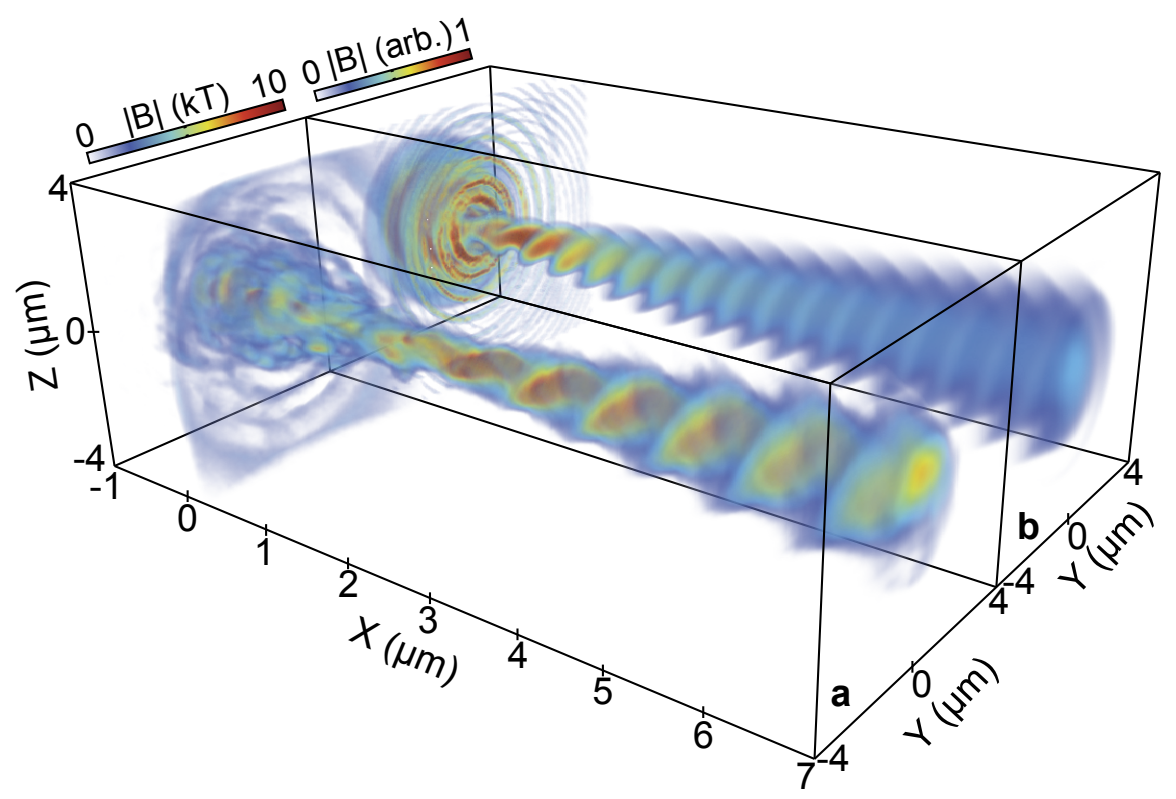

Figure 6: Magnetic field structure driven by circularly polarized light. a, Magnitude of the magnetic field driven by a circularly polarized laser pulse producing a relativistic plasma aperture in a $10 \mathrm{~nm}$-thick Al target. A helical field structure is produced. b, Same for the case of a lower intensity laser pulse propagating through a fixed, predefined aperture of $0.75 \mu \mathrm{m}$ radius, i.e. without plasma effects. The $3 \mu \mathrm{m}$ (FWHM) laser focus is centred on $[\mathrm{X}, \mathrm{Y}, \mathrm{Z}]=[0,0,0]$ in both cases and the field is sampled $30 \mathrm{fs}$ after the peak of the laser intensity. Diffraction induced by the aperture results in a helical field profile in both cases. The structure is modified by the presence of plasma in $\mathbf{a}$. 